\title{
The Height Measurement of Exposed Ball in Ball Pen Based on Machine Vision
}

\author{
Xu Qingqing ${ }^{1,}$, , Chen Fen ${ }^{1, b,{ }^{*}, \text { Guo Hengchang }}{ }^{2, c}$ \\ ${ }^{1}$ School of Economics and Management, Nanjing University of Science and Technology, \\ Nanjing, Jiangsu, China \\ ${ }^{2}$ M\&G Chenguang Stationery co., Ltd, Shanghai, China \\ axqq ing@163.com, ${ }^{b}$ chenfen@njust.edu.cn, ${ }^{c}$ hcguo@mg-pen.com \\ * Corresponding Author: Chen Fen, Associate Professor, chenfen@ njust.edu.cn
}

Keywords: Ball pen, Size measurement, Corner detection, Least square curve fitting

Abstract. The height measurement of exposed ball is one of the most important quality test items in the production of ball pens. The automatic detection method based on machine vision has huge superiority, compared with the traditional manual inspection method. In order to realize automatic measurement of exposed ball's height, image processing methods are introduced (e.g., corner detection and least-squares curve fitting) to develop the automatic detection system of exposed ball based on Halcon. The test results show that, compared with the traditional manual inspection, our proposal not only can ensure the detection accuracy, but also improve the detection efficiency.

\section{Introduction}

That "The ball of ball pen still need to be imported" said by Chinese Premier Li Keqiang has aroused great reflection on Chinese manufacturing, and people are amazed that the annual production of ball pens in China is about 40 billion, but the core technology and materials of Chinese pen industry are highly dependent on imports. It makes the pen industry attach more importance to cultivating its capacity of independent research and development, and the automatic inspection technology is one of the most important research.

The ball pen is widely used because of its simple structure, convenient carrying and fluent writing, etc. As one of the core components, the size of pen point need to be strictly controlled. The height of exposed ball (as shown in Fig. 1) is one of most important parameters in pen point because it has a great impact on pen's writing fluency and ink. While the production of pen points must go through many procedures, influenced by the machining accuracy, the exposed ball's size often does not meet the standard requirement. Therefore, it is necessary to measure the size of exposed ball before putting the pen into use, so as to eliminate unqualified pen points.

At present, the measurement of exposed ball's size is generally detected by the projector, microscope or other tools. Due to the operators' subjective factors, this manual inspection method often results in low accuracy and low efficiency. In addition, quantities of pen point to be detected is huge, while the manual inspection is time-consuming, so the speed of detection is difficult to meet the demand for the development of modern pen industry. However, with the advantages of non-contact, high precision, high flexibility and fast speed, the machine vision has been widely used in size measurement ${ }^{[1]}$. This makes it possible to measure the exposed ball's height automatically. In this paper, we will do some basic manipulation on the images captured, then use corner detection, least square curve fitting and other methods to develop an automatic inspection technology for measuring exposed ball's height based on Halcon.

\section{Overall Framework}


The specific process of this automatic size measurement is shown in Fig. 2. Firstly, we use image filtering and image enhancement to process the captured image. Secondly, improved Harris is applied to detect corners in images to select the desired corners (point A and point B as shown in Fig. 1). Thirdly, we extract the image edge, segment the edge into several contours, select the circular arc from the contours, and fit the circular arc with the least square so as to get its center (point $\mathrm{C}$ as shown in Fig. 1) and radius of the fitting circle. Finally, the distance $d$ from the center of the circle to the straight line (line AB as shown in Fig. 1) is calculated, and then combined with the radius $R$ to get the exposed ball's height $L=R-d$.

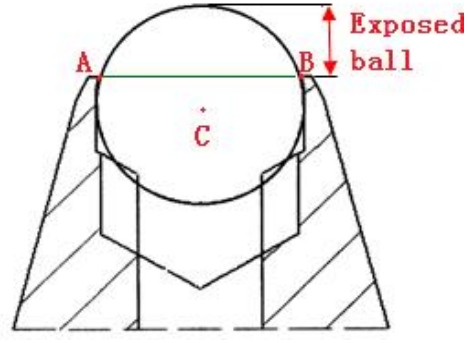

Fig. 1 Pen point structure

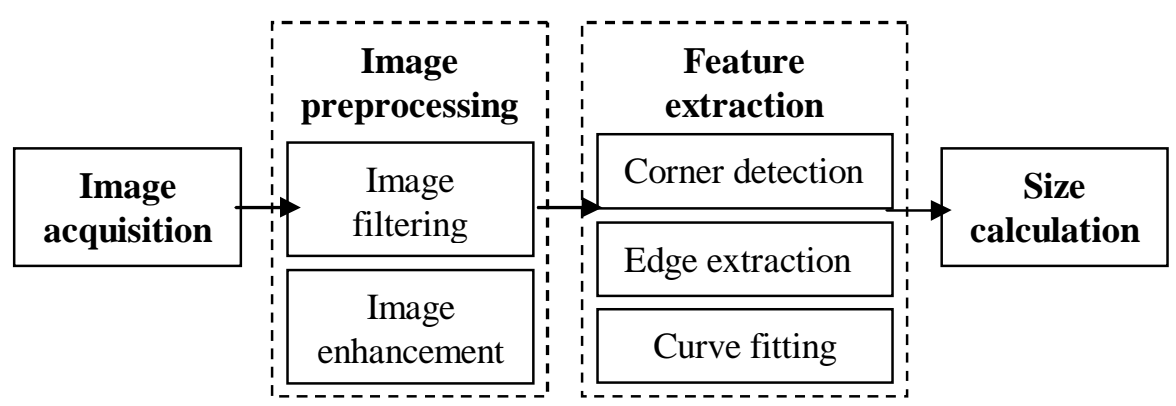

Fig. 2 Detection process

\section{Image Preprocessing}

In order to make the image features more obvious, we need to preprocess the image ${ }^{[2]}$, which is useful for the subsequent processing (feature extraction and target size calculation). In the process of image acquisition, the image will inevitably be influenced by various noise, resulting in the image quality degradation, which affects the whole effect of the image ${ }^{[3]}$. Therefore, first of all, the image need to be processed by filtering so as to eliminate or reduce noise. After a large number of experiments, the median filtering is used in this study, which not only can remove noise, but also effectively preserve the image edge. However, the image edge will become fuzzy after filtering, so it's necessary to enhance image contrast in order to reduce the negative effects and make the edge clear ${ }^{[4]}$.

The results of image preprocessing after median filtering and image enhancement are shown in Fig. 3. It shows that isolated noise points in original image can be effectively eliminated by median filtering, and the overall contrast of image is improved after image enhancement, and the edge becomes more prominent, which is conducive to target feature extraction.

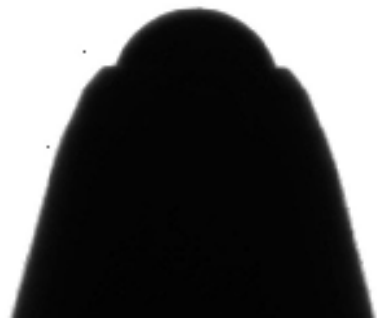

(a) Original image

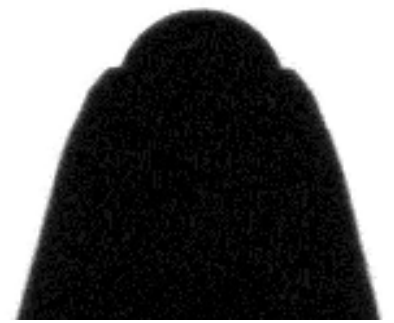

(b) Median filtering

Fig. 3 Image preprocessing

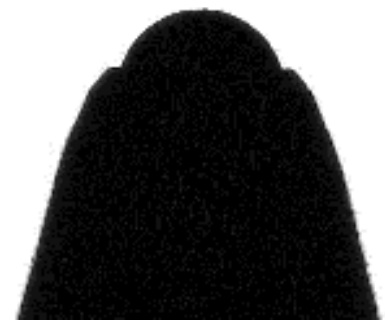

(c) Image enhancement

\section{Feature Extraction}

Image features are the basis of the image analysis, which determines the success of the algorithm. Corner and edge features are involved in this paper.

Corner Detection. As a stable and sparse feature, corners or feature-points contain very significant structural information in the image, which plays a key role in computer vision and image processing ${ }^{[5]}$. From the pen structure, we can find that corner detection is the most important step in the auto-measurement of exposed ball. Harris algorithm ${ }^{[6]}$ is adopted in our proposal.

The main idea of Harris corner detection algorithm is as follows: 
1) Construct the local auto-correction function $M$ by calculating image gradients $I_{x}$ and $I_{\mathrm{y}}$ in $\mathrm{x}$ and y directions and using the Gaussian function $w(x, y)$ to smooth image. The function $M$ is:

$$
M=\sum_{x, y} w(x, y)\left[\begin{array}{cc}
I_{x}^{2} & I_{x} I_{y} \\
I_{x} I_{y} & I_{y}^{2}
\end{array}\right]
$$

2) Calculate the eigenvalues $\lambda_{1}$ and $\lambda_{2}$ of matrix $M ; \lambda_{1}$ and $\lambda_{2}$ will be proportional to the principal curvatures of local auto-correction function; If both curvatures are high, this indicates a corner;

3) Calculate the corner response function $R$ according to the eigenvalues $\lambda_{1}, \lambda_{2}$ and the inhibition constant $k$. When $R$ of one point exceeds a predetermined threshold, this point will be viewed as a corner. The corner response function $R$ is:

$$
R=\operatorname{det} M-k(\text { trace } M)^{2}=\lambda_{1} \lambda_{2}-k\left(\lambda_{1}+\lambda_{2}\right)^{2}
$$

Halcon provides two Harris operators points_harris and points_harris_binomial, and the latter is an improved Harris operator, which can make the locations of corners more accurate so as to get the sub-pixel points. In this paper, we set $k=0.04$ and the threshold $T=1000$, and use the two operators to detect corners in the target image. Then, we need to select the only two points needed in size measurement from all corners obtained. Fig. 4 is the result of corner detection. As shown in Fig. 4, the locations of points detected by the original Harris operator have a little deviation, which is not accurately located on the image edge; in comparison, the improved Harris operator can make the locations of points more accurately.

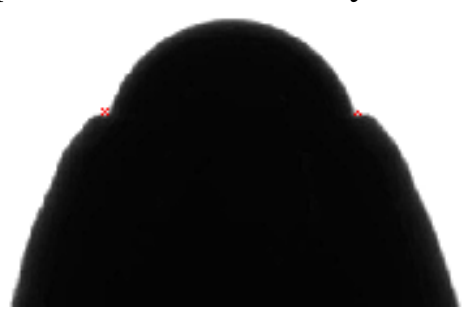

(a) Harris corner detection

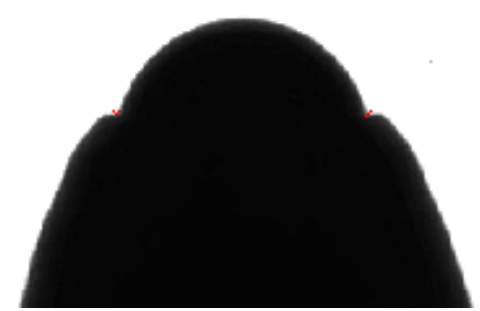

(b) Improved Harris corner detection

Fig. 4 Corner detection

Target Edge Extraction. The image edge is a region where the brightness of the image changes significantly, in other words, it is a buffer area where gray value changes a lot ${ }^{[7]}$. This paper uses edges_sub_pix operator to extract the sub-pixel edge by the canny filter. The comparison between pixel edge detection and sub-pixel edge detection is shown in Fig. 5. The red line represents sub-pixel edge, and the green one is pixel edge. We can see that the sub-pixel edge detection makes the adjacent two pixels subdivided into smaller units, which makes the extracted edge more precise.

After extracting the image edge, we need to get the target circular arc:

1) Segment the image edge. The segment_contours_xld operator in Halcon can segment the edge into line segments and circular or elliptic arcs.

2) Union circular arcs. The target circular arc may be split into several pieces in the process of edge segmentation, so the union_cocircular_contours_xld operator is used to merge all contours that belong to the same circle in order to make the target circular arc as complete as possible.

3) Calculate the circularity of each contour. The aim is to select the contour with the largest circularity as the target circular arc. Howerver, some contours affected by noise may have larger circularity, so it's essential to choose contours involved in circularity calculation according to their shape features (e.g., area and total length) before the calculation.

After the above steps, the target circular arc is shown in Fig. 6. 


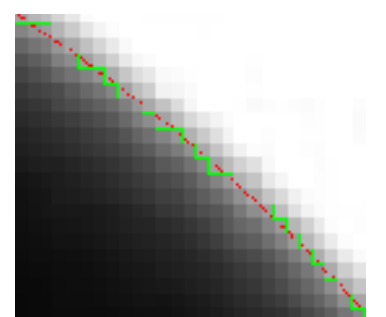

Fig. 5 Sub-pixel and pixel edge

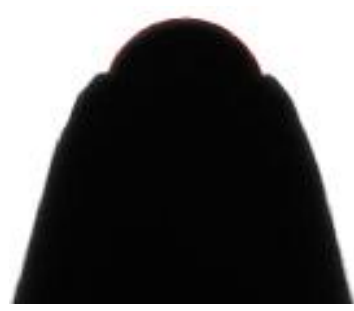

Fig. 6 Target circular arc

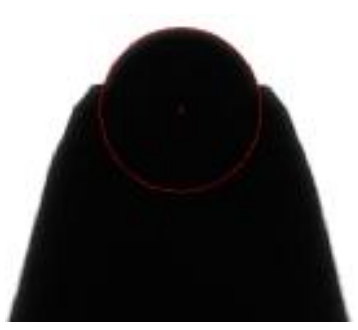

Fig. 7 Curve fitting

Curve Fitting. The purpose of extracting the target circular arc is to approximate the arc by a circle so as to get its center and radius. The least square method is applied to fit the pixels on the arc, whose main idea is to minimize square sum of distances between the contour points and the resulting circle ${ }^{[8]}$. The fit_circle_contour_xld operator is used for the fitting of the circle in this paper, and the result is shown in Fig. 7.

\section{Results and Discussion}

Size Calibration. After above processing, the pixel size of exposed ball is obtained. In order to gain the actual size of the object, it is necessary to establish the scaling relation between the pixel and the physical size, that is, the actual physical size of each pixel need to be calibrated ${ }^{[9]}$. This article uses the ratio calibration to obtain calibration coefficient $k$.

The calibrate method is described as follows:

1) Use the micrometer to measure the axial width $D$ (as shown in Fig. 8) of the pen point.

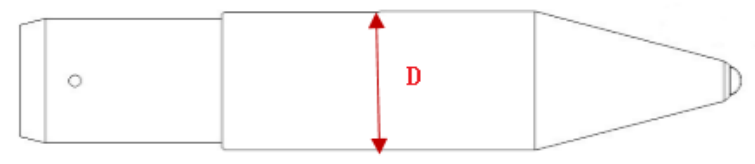

Fig. 8 Axial width of pen point

2) Ensure the environment (relative position of all the hardware, camera parameters, source etc.) of detection system is basically consistent with the exposed ball to be detected, then use algorithms of image processing to calculate the pixel size $d$ of axial width. The calibration coefficient $k=\frac{D}{d}$.

To make the calibration coefficient more accurate, we carry out multiple calibrations with different pen points, then take the average value as the final calibration coefficient, finally $k=0.00184 \mathrm{~mm} / \mathrm{pixel}$.

Experiments. The size standard for the exposed ball is $0.17_{-0.01 \mathrm{~mm}}^{+0.02 \mathrm{~mm}}$ in the production of pen point, namely, if the measured size is greater than $0.19 \mathrm{~mm}$ or less than $0.16 \mathrm{~mm}$, this pen point will be regarded as an unqualified product. In order to verify the detection accuracy, the automatic detection method is compared with the manual inspection method with projector (that is the measurement tool used by the enterprise). We measure the exposed ball's size of 200 pen points with these two methods in our experiment, and the measurement results of 20 groups of data among them are illustrated in Table 1. The experimental results show that relative errors between two methods are within $\pm 5 \mu \mathrm{m}$, which can meet the required measurement accuracy of the enterprise.

Table 1 Comparison of measurement results

\begin{tabular}{cccc|cccc}
\hline NO. & $\begin{array}{c}\text { Manual } \\
\text { method/[mm }]\end{array}$ & $\begin{array}{c}\text { Automatic } \\
\text { method/[mm }]\end{array}$ & $\begin{array}{c}\text { Relative } \\
\text { error/[mm }]\end{array}$ & NO. & $\begin{array}{c}\text { Manual } \\
\text { method/[mm }]\end{array}$ & $\begin{array}{c}\text { Automatic } \\
\text { method/[mm }]\end{array}$ & $\begin{array}{c}\text { Relative } \\
\text { error/[mm }]\end{array}$ \\
\hline 1 & 0.169 & 0.171 & -0.002 & 11 & 0.169 & 0.167 & 0.002 \\
2 & 0.175 & 0.174 & 0.001 & 12 & 0.167 & 0.166 & 0.001 \\
3 & 0.176 & 0.177 & -0.001 & 13 & 0.169 & 0.169 & 0.000 \\
4 & 0.174 & 0.176 & -0.002 & 14 & 0.173 & 0.170 & 0.003 \\
5 & 0.168 & 0.167 & 0.001 & 15 & 0.170 & 0.168 & 0.002 \\
6 & 0.171 & 0.174 & -0.003 & 16 & 0.168 & 0.168 & 0.000 \\
\hline
\end{tabular}




\begin{tabular}{cccc|cccc}
\hline 7 & 0.174 & 0.174 & 0.000 & 17 & 0.170 & 0.172 & -0.002 \\
8 & 0.170 & 0.172 & -0.002 & 18 & 0.170 & 0.168 & 0.002 \\
9 & 0.173 & 0.173 & 0.000 & 19 & 0.170 & 0.169 & 0.001 \\
10 & 0.171 & 0.170 & 0.001 & 20 & 0.169 & 0.171 & -0.002 \\
\hline
\end{tabular}

In addition, we statistics the accuracy rate (as shown in Table 2) which means the ratio of the number of samples detected correctly by our proposal to the total sample. It can be seen that accuracy rate is $97.5 \%$ and the average time spent in measuring one image by the automatic detection method is less than $300 \mathrm{~ms}$, which has greater superiority in detection rate compared with the manual method.

Table 2 Statistical results

\begin{tabular}{cccccc}
\hline $\begin{array}{c}\text { Sample } \\
\text { number }\end{array}$ & $\begin{array}{c}\text { Accurate } \\
\text { number }\end{array}$ & $\begin{array}{c}\text { Misjudged } \\
\text { number }\end{array}$ & $\begin{array}{c}\text { Accuracy } \\
\text { rate/[\%] }\end{array}$ & $\begin{array}{c}\text { Average } \\
\text { error/[mm }]\end{array}$ & $\begin{array}{c}\text { Average detection } \\
\text { time/[ms }]\end{array}$ \\
\hline 200 & 195 & 5 & 97.5 & 0.0028 & 285 \\
\hline
\end{tabular}

\section{Conclusions}

In this paper, the machine vision technology is applied to the measurement of the exposed ball' height in pen point. We propose an auto-measurement method by image processing technology such as filtering, enhancement, corner detection and least square curve fitting. Finally, the automatic measurement results are compared with the traditional manual measurement results. The experimental results show that automatic measurement method based on machine vision not only can be more convenient and fast, but also reach the required precision of size measurement, which can meet the development requirements of modern pen industry.

\section{Acknowledgements}

This paper is supported by the Specific Funds of Shanghai Municipal Commission of Economy and Informatization for Industrial Restructuring and Upgrading "Research on Automatic Inspection Technology and its Application in the Pen Industry based on Machine Vision" (No.: Shanghai CXY-2016-009).

\section{References}

[1] Li Xiaowei. Research on Key Technology of Precision Measurement System for Geometrical Sizes of Chip Components [D]. Chongqing University, 2014.

[2] Zhou Chunxia, Wei Min, Tang Zhengning. Printing Dot Image Segmentation Method Based on the Mathematical Morphologic [J]. Packaging Engineering, 2007, 28(6):14-15.

[3] Xu Erning. The Development of the Small Part Dimension Detection System Based on Image Processing Technology [D]. East China University of Science and Technology, 2014.

[4] Yang Zongfang, Bai Jianyu. Vial Size Detection Based on Machine Vision [J]. Computer Measurement \& Control, 2016, 24(8):63-66.

[5] Zhang Weichuan, Kong Xiangnan, Song Wen. Review of Image Corner Detection Algorithms [J]. Acta Electronica Sinica, 2015, 43(11):2315-2321.

[6] Harris C, Stephens M. A combined corner and edge detector[C]. Alvey vision conference. 1988, 15(50): 147-151.

[7] Xu Zhenying, Xu Jiaxiang, Huang Jianbin, et al. Fast Precision Measurement of the Apertures of Electric Connector Shell Based on Halcon [J]. Manufacturing Automation, 2015, 37(22):31-33. 
[8] Fu Gui, Li Ketian, Li Yang. Research on the Measure System of Light Guide Plate Mould Based on Machine Vision [J]. Manufacturing Technology and Machine tools, 2016(10):58-61.

[9] Hao Yongping, Wang Yongjie, Zhang Jiayi, et al. Pixel Equivalent Calibration Method for Vision Measurement [J]. Nanotechnology and Precision Engineering, 2014, 12(5):373-380. 